\title{
Integrated System for Robot-Assisted in Prostate Biopsy in Closed MRI Scanner
}

\author{
Philip W. Mewes, Junichi Tokuda, Simon P. DiMaio, Gregory S. Fischer, Csaba Csoma, David G. Gobbi, \\ Clare M. Tempany, Gabor Fichtinger, Nobuhiko Hata, Member, IEEE
}

\begin{abstract}
Prostate cancer biopsy is a routine medical procedure, yet conventional techniques suffer from low sensitivity attributed to suboptimal image guidance and needle placement. Targeting small lesions and foci $(5 \mathrm{~mm}$ in diameter) is particularly prone to errors. We developed an integrated system to perform robot-assisted transperineal needle insertions into the prostate, under Magnetic Resonance Imaging (MRI) guidance. The system provides arbitrary needle trajectories and allows for simultaneous surveillance and correction of the needle path, based on intra-operative MRI. System functionality and data transfer and processing tests were conducted. Five lesions embedded in the gel phantom were targeted successfully, while communication delays (due to higher image frame rates) had no adverse affect on robot-software communication. The system was sufficiently resistant to high network loads and performed with an acceptable transfer rate.
\end{abstract}

\section{INTRODUCTION}

With an estimated 220,900 new cases in 2003, prostate cancer is the second most common cancer in men in the United States, and is responsible for about 33,000 deaths annually [1]. Indeed, prostate cancer accounts for $33 \%$ of all malignancies in men, has the highest lifetime probability of occurrence ( 1 in 6 men), and one of the highest death rate and mortality volumes among cancer. About $1.5 \mathrm{~m}$ prostate cancer biopsies are performed each year in the U.S. alone. At the same time, the sensitivity of current diagnostic methods involving palpation, serum Prostate-Specific Antigen (PSA) and ultrasound-guided biopsy are limited. In prior work at the Brigham and

Manuscript received September 14, 2007. This publication was made possible by Grant Numbers 5U41RR019703, 5P01CA067165, 1R01CA111288 from NIH. Its contents are solely the responsibility of the authors and do not necessarily represent the official views of the NIH. This study was also in part supported by NSF 9731748 and CIMIT.

PW Mewes ,J Tokuda, C. Tempany, and N. Hata are with the Brigham and Women's Hospital, Department of Radiology, 75 Francis St. Boston, MA 02115, USA (phone: 617-732-5809; fax: 617-582-6033; e-mail: [pmewes, tokuda, ctempany, hata]@bwh.harvard.edu ).

S DiMaio is with Intuitive Surgical Inc., 950 Kifer Road, Sunnyvale, California, 94086, USA (phone: 408-523-2309 fax: 408-523-1390).

GS Fischer and C Csoma are with the Johns Hopkins University, Center for Computer Integrated Surgery, 3400 N. Charles St., Baltimore, MD 21218, USA (phone: 410-516-4723; fax: 410-516-3332; e-mail: [gfischer, csoma]@jhu.edu .

DG Gobbi and G Fichtinger are with the School of Computing at Queen's University, 25 Union Street, Kingston, Ontario, K7L 3N6, Canada (phone: 613-533-6050; fax: 613-533-6513; e-mail: [dgobbi, gabor]@cs.queensu.ca).
Women's Hospital, we have developed an MRI-guided transperineal prostate biopsy approach that allows for targeted sampling of suspected abnormalities. Our clinical experience from 53 cases has shown that MRI-guided prostate biopsy has a greater sensitivity than ultrasoundguided biopsy, owing to the superior prowess of MRI in showing suspicious lesions and foci [1]. While the use of MR imaging appears to have improved clinical outcomes, conventional manual needle placement was found to be a major limitation. First, modern high field closed scanners do not allow access to the patient inside the magnet. The subject must be translated in and out of the scanner, which raises the probability and extent of target dislocation. The is use of conventional fixed needle guide templates limits targeting resolution and trajectory [1],[2]. To overcome these limitations, we have been investigating the use of remotely actuated mechanical assistants and robots [4-9], toward achieving targeting and needle insertion inside the magnet, under real-time MR imaging.

In this paper, we present a system integration strategy, architecture, and integration of MRI imaging, robot control, target and trajectory planning and process monitoring. We report the results of phantom experiments in closed bore $3 \mathrm{~T}$ MRI scanner.

\section{II.METHODS}

The architecture of the proposed system is outlined in Figure 1. The following core and subsystems have been used to achieve semi-automatic needle-based interventions.

\section{A. 3D Slicer for control, planning and visualization}

We developed an add-on software module for the open source software 3D Slicer [3]. This serves core software for control, planning and visualization. 3D Slicer is a flexible research platform providing an arsenal of functions for medical image processing, visualization and surgical navigation with integrated graphical user interface (GUI). Our add-on module to Slicer provides connectivity with the MRI scanner and robot, and allows the operator to monitor and control the subsystems from the GUI of 3D Slicer.

The following functions were implemented in the prostate intervention module:

- Status management: Control and switch status of subsystems is provided, based on current work phase. 


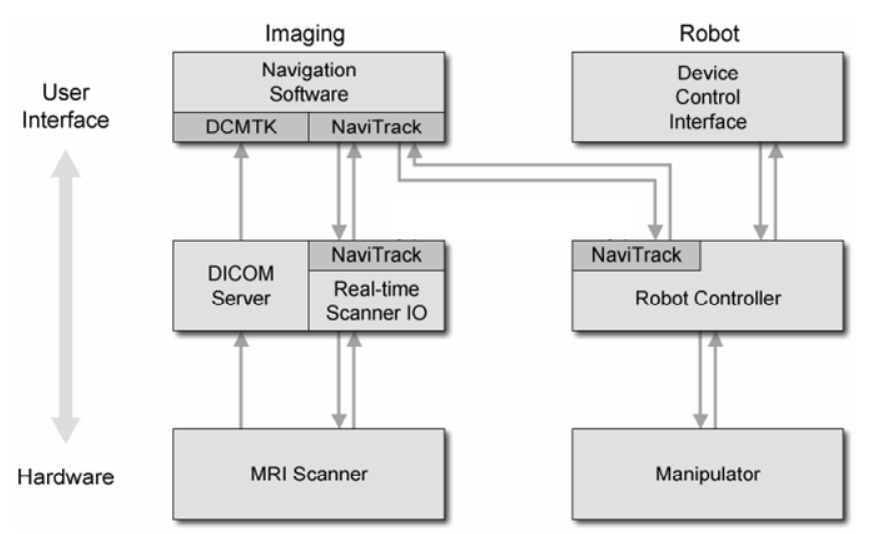

Fig. 1: The diagram shows overview of the developed integrated MRI and robot control software system for prostate intervention. The navigation software, scanner input-output (IO) and robot controller are connected using the NaviTrack framework for bi-directional data stream.

- Dynamic Network configuration: Connections among subsystems are set via configuration file.

- Scanner Control: The operator starts, stops, or pauses scanning through the module's GUI.

- Real-time Scan Plane Control: MRI scan plane is set dynamically to provide optimal view of the needle, calculated from current needle position.

- Visualization of Needle Trajectory: The predicted needle trajectory is superimposed on the MRI images, in both $2 \mathrm{D}$ and $3 \mathrm{D}$ views.

- Target Points Managements: The operator can create and manage a list of targets and associated trajectories.

- Manual Robot Control Interface: Movement of the robot can be controlled manually

- Real-time Imaging: The dynamically controlled 2D real-time MRI image is displayed in the 3D viewer.

\section{B. NaviTrack for communication}

We adapted NaviTrack for network communication [4]. NaviTrack is multi-modal software to transfer intraoperative image, current position and orientation information of the robot and control commands for the scanner and the robot. NaviTrack works as an interface for each subsystem to the network, as shown in Fig. 1. Dataflow in the network can be defined in a configuration file described in extensible markup language (XML), allowing the user to change the structure of the network. NaviTrack is also in charge of preprocessing the MRI images coming from the scanner and robot position data coming from the robot.

\section{MRI-compatible robot}

The system incorporates an MR-compatible robot with 4 degrees of freedom (DOF) [5], providing vertical planar motion (100 $\mathrm{mm} \max )$, positive elevation angle $\left(15^{\circ} \mathrm{max}\right)$, horizontal planar motion ( $\pm 50 \mathrm{~mm} \max )$ and azimuth angle $\left( \pm 15^{\circ}\right.$ max.) A guide sleeve is mounted on the robot to provide encoded needle insertion ( $120 \mathrm{~mm}$ max), manually under direct control of the physician. For position sensing the robot is using MR-compatible linear optical encoders, which allows tracking position and orientation of the robot's end effector.

\section{MRI Scanner}

A 3T whole body MRI scanner (Signa HDx, GE Healthcare, Chalfont St. Giles, Buckinghamshire, United Kingdom) is used to acquire MR images before and during the procedure. The NaviTrack middleware is installed in the host workstation of the scanner system to control the imaging plane, based on the input from 3D Slicer. During real-time imaging, raw images are transferred to 3D Slicer through the network on-the-fly.

\section{E. Z-Frame for scanner-to-robot registration}

Before use, the robot must be registered to the coordinate frame of the MRI scanner. For this purpose, seven fiducial markers are arranged in a Z-shaped configuration on three sides of a cube, and attached to the robot (Fig.2), adopting

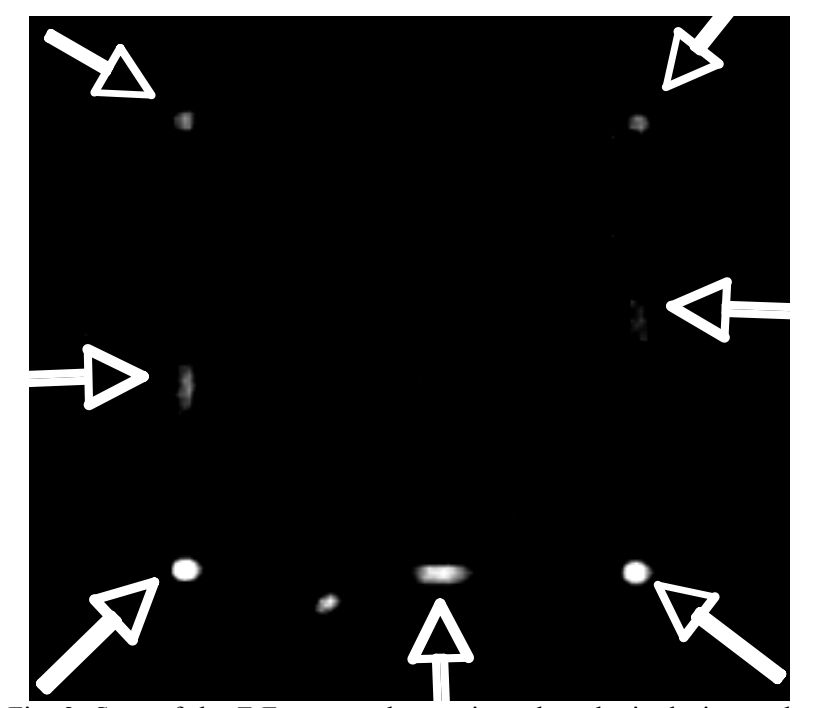

Fig. 3: Scan of the Z-Frame used to register the robotic device to the patient coordinate system. Notice the arrows which mark the four corners and three marks showing a crop of the diagonals of the frame.

from our prior work [6]. The Z-frame consists of acrylic plates and columns with integrated horizontal and diagonal glass tubes filled with an MR-visible contrast agent solution in water (Fig. 2). The dimensions and constellation of the Z-shapes, relatively to the robot are known by precise manufacturing. From the intersection points of a single 2D MR image with the Z-frame (Fig. 3), we determine the relative pose of the Z-frame relative to MRI. The closed form registration algorithm is implemented in the module in $3 \mathrm{D}$ Slicer. The calculated pose of the Z-Frame is sent to the robot controller upon request.

\section{F. Workflow}

The behavior of each subsystem is determined by current state. Six states are defined in the clinical workflow: Start Up, Planning, Calibration, Targeting, Manual and Emergency. 3D Slicer invokes state transitions by sending 
work-phase transition commands to the subsystems through the NaviTrack connection.

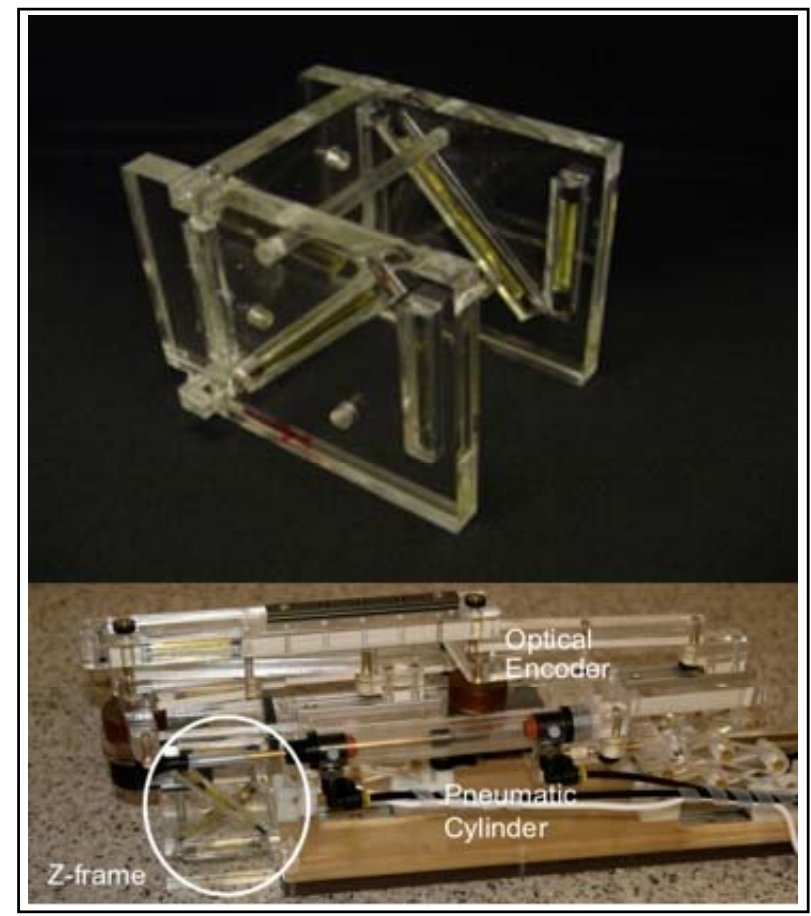

Fig.2: Z-Frame cube and MR-compatible robot with the attached $Z$-Frame cube on the front.

\section{EXPERIMENTS}

Two sets of experiments of system functionality and data transfer were conducted. The primary goal of these tests was to demonstrate that our software structure is able to execute the complete chain of commands and actions required. The secondary goal was to test the ability of handling high frequency and bandwidth transfer of image and position data.

\section{A. System Functionality Test}

To simulate transperineal prostate biopsy, we used a silicon phantom representing the prostate and embedded lesions.

First, we tested the connection between all components and particularly the ability of querying the active states of the scanner and the robot. This test was necessary to guarantee the correct order of steps and general safety of the procedure.

Second, we simulated treatment planning, by localizing several lesions in a high resolution MRI volume image planning the optimal trajectory by picking perineal entry points. In actual clinical cases, diagnostic-quality images are acquired several days before the intervention to identify target locations and registered to an MRI volume image acquired intra-operatively [2]. In this experiment, 3D image was acquired with a 3D fast spoiled gradient recalled (SPGR) sequence to image the target region $(\mathrm{TR}=7 \mathrm{~ms}, \mathrm{TE}=2 \mathrm{~ms}$, flip angle $=30 \mathrm{deg}, \mathrm{FOV}=200 \mathrm{~mm}$, slice thickness $=5 \mathrm{~mm}$, axial acquisition, image matrix 256×256 pixels). An 8-channel TORSO body coil was used for the image acquisition. The trajectory of the needle for each lesion was then preset by adjusting the orientation of the robots end-effector.
Third, the robotic device was registered to the MRI coordinate system by using a single image plane intersecting the Z-Frame. Images were acquired after scanner and phantom were placed inside the MRI scanner. The Z-Frame position was calculated in 3D Slicer and transferred to the robot controller. To acquire images from the Z-Frame we used a $2 \mathrm{D}$ fast $\mathrm{SPGR}$ sequence $(\mathrm{TR}=34 \mathrm{~ms}, \mathrm{TE}=3 \mathrm{~ms}$, axial acquisition, $\mathrm{FOV}=160 \mathrm{~mm}$, image matrix 256x256 pixels).

Next, targeting and needle insertion was performed to reach the target locations identified in the phantom. Targeting was initiated by sending the coordinates and desired trajectory of each lesion to the robot. During the alignment of the needle, 3D Slicer was used to control the acquisition of a

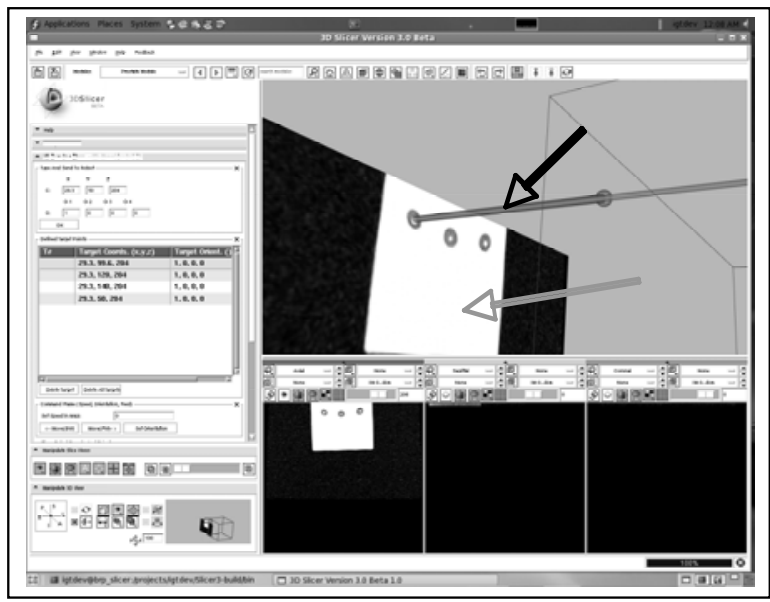

Fig. 4: Visualization of the biopsy needle (indicated by the black arrow) in 3D Slicer during the insertion process into a silicon phantom (indicated by the grey arrow). After the needle reached its target the biopsy gun is fired or the radiation probe is placed inside the lesion. The needle was then retracted. The last two work steps a repeated for every target point.

real-time (RT) image from the scanner, showing the actual position of the needle in a sagittal or coronal scan plane (Fig. 4). We used 2D fast gradient recalled echo (FGRE), with the same parameters as for imaging the Z-Frame, except the FOV was increased to $200 \mathrm{~mm}$. Upon confirming correct needle position, the physician started the insertion process, while continuously verifying the needle position and trajectory on the real-time image (Fig. 4). This was achieved by reaching inside the magnet and manually advancing the needle inside the guide sleeve. During insertion, the robot's optical encoders were sending information about the needle's position and orientation through the robot controller to 3D Slicer. Based on this information, the scanner was controlled automatically to change scanning position, to acquire an optimal image of the needle. The actual orientation of the needle was verified relative to the planned orientation, and then it was maintained during insertion process. In the follow-up only the feed of the needle towards the target was adjusted manually. To verify the correct conversion of our targeting commands a 3D volume confirmation scan was acquired to verify the location of the needle visualization with respect to the actual needle artifact. 


\section{B. Data Transfer Test}

We measured the delay between the subsystems (robot and scanner) and 3D Slicer. To avoid measurement errors due to difficult synchronization of different processor-clocks of the subsystems, we analyzed the relative value of the delay based on the smallest obtained value. We assumed that the frame rate of the real-time (RT) imaging is between 0.2 and 0.5 frames per second (FPS) and that position of the robot is monitored every $100 \mathrm{~ms}$. This part of the experiment is to show how the different image transfer rates affect the robot transfer rate.

\section{RESULTS AND DISCUSSION}

Graphical feedback from 3D Slicer software and direct MR confirmation scans both showed that the position of the needle tip was correctly transferred to the robot controller. This test was repeated for five targets situated at different insertion depths.

Table 1 shows the data transfer delay between the host workstation of the Scanner and 3D Slicer each time a RT

TABLE 1

MEAN AND STANDARD DEVIATION OF ROBOT AND SCANNER COMMUNICATION DELAY WITH 3D SLICER VARIED BY DIFFERENT IMAGE FRAME RATES FOR REAL-TIME VISUALIZATION.

\begin{tabular}{lcl}
\hline $\begin{array}{l}\text { Frame rate } \\
\text { (fps) }\end{array}$ & $\begin{array}{l}\text { Robot delay } \\
\left.\text { (zero-referenced }{ }^{*}, \mathrm{~ms}\right)\end{array}$ & $\begin{array}{l}\text { Scanner delay } \\
(\mathrm{ms})\end{array}$ \\
\hline 0.2 & $0 \pm 32$ & $92 \pm 29$ \\
0.25 & $98 \pm 31$ & $73 \pm 27$ \\
0.3 & $220 \pm 21$ & $61 \pm 28$ \\
0.5 & $240 \pm 26$ & $49 \pm 27$ \\
\hline \hline * The smallest delay of the measurement was taken as the reference for \\
the following test series. Consequently to our results the smalls delay \\
was found in the robot-to-3DSlicer-communication using the smallest \\
applied real-time frame rate.
\end{tabular}

image is sent and the delay between the Robot Controller and $3 D$ Slicer every time a position dataset was sent, as observed during needle positioning and subsequent insertion.

As seen in Figure, 1 the robot controller and the scanner using the NaviTrack framework to communicate with 3D Slicer. Each NaviTrack packet contains the time stamp when it was sent. On the receiving end (3D Slicer computer) we subtracted this timestamp from the current machine's time by implemented a $\mathrm{C}++$ routine using gettimeofday() functions. The smallest value becomes the "zero delay reference".

The difference between the reference value and subsequent measurement values were assumed to be caused by network delays. Table 1 shows the relative differences between the timestamps on the planning workstation (that runs 3D Slicer) and the timestamps on the MR scanner and Robot Controller.

Communication delay between the MR scanner and 3D Slicer increased with higher frame rates, which was not particularly surprising as the reception of the incoming MR image takes considerable time and it is easily outpaced by querying the robot position.

Table 1 indicates increasing time delay due to the reception and processing of the real-time MR images coming from the scanner, as compared to the delay caused by the robot communication. The delay caused by communication with the robot was due to position information that 3D Slicer received from the scanner to visualize the needle position. This delay is negligible compared to the delay resulting from the image transfer.

In conclusion, the software framework showed potential to address several shortcomings of conventional manual needle placement in MRI-guided prostate biopsy. We were successful in executing a complex clinical workflow. Compared to conventional manual template-based methods, we demonstrated the function of continuum needle spacing in arbitrary trajectory. We accomplished targeted needle placement under RT image surveillance, reliably, repeatedly, and without apparent holdup of the normal clinical workflow. We successfully integrated feedback from the robot into the workflow of the intervention to visualize, monitor and verify the movement of the robot and the position needle tip.

Our results show that (1) the software framework is sufficiently resistant to high network loads, (2) the system performed with an acceptable transfer rate, (3) it can robustly handle several different intercommunicating subsystems, and (4) 3D Slicer kept up with the incoming flux of data.

Although our motivating application is prostate biopsy, the proposed architecture is directly applicable to MRI-guided localized prostate cancer therapies, such as brachytherapy and it is also adaptable to a variety of MRI-guided needle placement procedures in other organ systems and diseases.

\section{REFERENCES}

M. J. So, S. Haker, K. H. Zou, A. Szot Barnes, R. Cormack, J. P. Richie, A. V. D'Amico, and C. M. Tempany, "Clinical Evaluation of MR-guided Prostate Biopsy," in ISMRM 13th Scientific Meeting and Exhibition, Miami, FL, 2005, p. 264.

E. Schneider, K. W. Rohling, M. D. Schnall, R. O. Giaquinto, E. A. Morris, and D. Ballon, "An apparatus for MR-guided breast lesion localization and core biopsy: design and preliminary results," J Magn Reson Imaging, vol. 14, pp. 243-53, Sep 2001. D. T. Gering, A. Nabavi, R. Kikinis, N. Hata, L. J. O'Donnell, W. E. Grimson, F. A. Jolesz, P. M. Black, and W. M. Wells, 3rd, "An integrated visualization system for surgical planning and guidance using image fusion and an open MR," J Magn Reson Imaging, vol. 13, pp. 967-75, Jun 2001.

G. Reitmayer and D. Schmalstieg, "OpenTracker - A Flexible Software Design for Three-Dimensional Interaction," 2.0.0 ed Vienna: Vienna University of Technology, 2001.

S. DiMaio, G. Fischer, S. Haker, N. Hata, I. I, C. M. Tempany, and G. Fichtinger, "A System for MRI-guided Prostate Intervention," in International Conference on Biomedical Robotics and Biomechatronics (BioRob) - Feb 2006 Pisa, Italy, 2006.

S. P. DiMaio, E. Samset, G. Fischer, I. Iordachita, G. Fichtinger, F. Jolesz, and C. M. Tempany, "Dynamic MRI scan plane control for passive tracking of instruments and devices," Med Image Comput Comput Assist Interv Int Conf Med Image Comput Comput Assist Interv, vol. 10, pp. 50-8, 2007. 